\title{
Experiencias de envejecimiento en el México rural
}

\author{
Sandra Treviño-Siller, Dra en Antrop,(I) Blanca Pelcastre-Villafuerte, Dra en Psicología Social,(I) \\ Margarita Márquez-Serrano, $M$ en Medicina Social.(1)
}

Treviño-Siller S, Pelcastre-Villafuerte B, Márquez-Serrano M. Experiencias de envejecimiento en el México rural. Salud Publica Mex 2006;48:30-38.

\section{Resumen}

Objetivo. Explorar la experiencia de envejecimiento de hombres y mujeres mayores de 60 años, que viven en contextos rurales de pobreza en los estados de Guerrero y Morelos, México. Material y métodos. Se diseñó un estudio cualitativo basado en entrevistas semiestructuradas. Se trabajó en comunidades con menos de 2500 habitantes, pobres y de alta marginación. Resultados. La experiencia de envejecimiento es radicalmente diferente según la condición de género; esta vivencia resulta ser más positiva para las mujeres. La soledad es un factor que configura la experiencia diferencial del envejecimiento, así como las redes sociales y el estado de salud-enfermedad. Los principales temores asociados a la vejez fueron la soledad, la enfermedad, la pobreza y la pérdida de independencia. Conclusiones. La identidad de género desempeña un papel fundamental en la configuración de la experiencia diferencial del proceso de envejecimiento, debido a que las oportunidades de desarrollo y las responsabilidades que se derivan de ella, promueven la desigualdad entre los sexos. Es necesario hacer más eficiente la respuesta institucional, de manera que responda a las necesidades específicas de esta población.

Palabras clave: envejecimiento; pobreza; identidad de género; México
Treviño-Siller S, Pelcastre-Villafuerte B, Márquez-Serrano M. Aging experiences in rural Mexico. Salud Publica Mex 2006;48:30-38.

\section{Abstract}

Objective. To explore the aging experience among men and women older than 60 years, living in rural areas from the states of Guerrero and Morelos, Mexico. Materials and Methods. A qualitative study was conducted in povertystricken, underserved communities with less than 2500 inhabitants. Semi-structured interviews were applied to collect data. Results. The aging experience is different according to gender, being more positive for women. Loneliness, together with social networks and the health-illness process, make the difference in the aging experiences. The main fears associated with aging are loneliness, illness, poverty, and loss of independence. Conclusions. Gender identity plays a central role in different aging experiences because the chances for development and accompanying responsibilities foster the inequality between men and women. It is necessary to improve institutional responses tailored to the specific needs of this population.

Key words: aging; poverty; gender; Mexico

Esta investigación fue parcialmente financiada por INDESOL y por el Fulbright New Century Scholars Program. En ambos proyectos la Dra.V. Nelly Salgado de Snyder fue la investigadora responsable.

(I) Dirección de Determinantes y Retos del Sistema de Salud, Centro de Investigación en Sistemas de Salud, Instituto Nacional de Salud Pública.

Fecha de recibido: 19 de octubre de 2004 • Fecha de aprobado: 24 de noviembre de 2005

Solicitud de sobretiros: Dra. Blanca Pelcastre-Villafuerte, Jefa de Departamento. Instituto Nacional de Salud Pública, Centro de Investigación en Sistemas de Salud, Dirección de Determinantes y Retos del Sistema de Salud. Av. Universidad No. 655,

Col. Sta. María Ahuacatitlán, Cuernavaca, Morelos, México.

Correo electrónico: bpelcast@correo.insp.mx 
E proceso de envejecimiento de la población es una realidad en el mundo y supone cambios con consecuencias estructurales, económicas y sociales nunca antes vistas en la historia de la humanidad. Las mejoras en los servicios de salud y las innovaciones tecnológicas en procesos de diagnóstico y tratamiento han permitido el incremento significativo en la esperanza de vida al nacer, a pesar de que todavía se observan diferencias por regiones, por nivel de desarrollo y por condición socioeconómica, de género y etnia. Así, a comienzos del siglo XX la población mundial, en promedio, podía esperar vivir alrededor de 40 años, mientras que al inicio del siglo XXI esa cifra se ha incrementado a 70 años. Datos recientes indican que para el año 2050 la población mayor de 60 años de edad se incrementará, de los 600 millones actuales a 2000 millones. $^{1}$

En algunos países desarrollados las pirámides poblacionales ya presentan cambios sustanciales en la base y en la cúspide y, en el caso de México, las proyecciones poblacionales indican que, para el año 2050, uno de cada cuatro mexicanos será mayor de 65 años de edad, pasando de los siete millones de adultos mayores en la actualidad a entre 27 y 32 millones. $^{2}$ Esto significa que, en México, el paisaje social cotidiano será diferente al actual y que, cada vez con mayor frecuencia, se convivirá entre generaciones con vivencias, necesidades y creencias diferentes, lo que constituye un verdadero reto en el ámbito social, político y cultural.

El envejecimiento es un proceso natural e inevitable; sin embargo, la forma como se envejece y el significado de este proceso son características que se construyen socialmente y varían de una cultura a otra. De forma similar, este proceso implica una serie de experiencias que son necesariamente diferenciadas, entre otros factores, por la condición de género, entendida como los roles y comportamientos culturalmente asumidos para hombres y mujeres. ${ }^{3,4}$ En el contexto mexicano, las identidades de género están enormemente arraigadas, sobre todo en el ámbito rural, donde los vínculos que se establecen entre las personas están mediados por los roles sociales que se derivan de esta identidad -las mujeres, como madres y amas de casa, es decir, como cuidadoras de otros; los hombres, como figuras de autoridad, fuerza y poder y como jefes de familia (proveedores) $)^{-5-7}$ mismos que se inculcan en la familia y en la sociedad desde la infancia.*

\footnotetext{
* Pelcastre B, Riquer F, De León V, Reyes H, Gutiérrez G, Bronfman M. ¿Qué se hace para no morir de hambre?: dinámicas domésticas y alimentación en la niñez en un área rural de extrema pobreza de México. En proceso.
}

Así, las personas asumen esta identidad y, a lo largo de su vida, desempeñan el papel que la misma sociedad les demanda y que los confirma como hombres o mujeres. ${ }^{8}$

La pobreza es otro factor que se asocia a las experiencias de envejecimiento; en México se estima que alrededor de un cuarto del total de la población vive en comunidades rurales ${ }^{9}$ y que cerca de 52.5 millones de personas son pobres, ${ }^{10}$ aunque existen variaciones en la estimación de este último dato en virtud de los diferentes enfoques con respecto a la medición de las líneas de pobreza. ${ }^{11-13}$

Independientemente de la forma que han utilizado para medir la pobreza, diversos autores coinciden en señalarla como una condición de carencia: de bienes, de necesidades satisfechas, de ingreso económico, de capacidades básicas para la autodeterminación, etcétera. La pobreza está vinculada con la desnutrición, el analfabetismo, la falta de autoestima y la imposibilidad de gozar de una salud aceptable, entre otros aspectos, ${ }^{14}$ de manera que este panorama de carencias generalizadas afecta, de manera inevitable, la experiencia de las personas en las diferentes etapas de la vida y, sobre todo, durante la vejez.

La situación de vulnerabilidad social y económica de la población adulta mayor se explica porque la mayoría ha dejado de estar inserta en el proceso laboral y/o no cuenta, necesariamente, con apoyos familiares, sociales o económicos suficientes, constantes y adecuados. ${ }^{15}$ De acuerdo con el Censo de 2000, $3.3 \mathrm{mi}-$ llones de ancianos, es decir siete de cada diez, viven en municipios de bajo o muy bajo desarrollo social; ${ }^{16} \mathrm{y}$ $36 \%$ de los hogares donde residían adultos mayores, según el Censo de 1990, no percibía ingresos. Esta situación es más drástica en contextos rurales donde, además, en muchas ocasiones la jefatura del hogar es femenina y no se cuenta con pensiones o son muy bajas: ${ }^{17}$ apenas $30 \%$ de los hombres y $15 \%$ de las mujeres reciben una pensión por jubilación o viudez; en el ámbito rural esas cifras descienden a $11.8 \%$ y $5.2 \%$, respectivamente. ${ }^{18-20}$ A todo lo anterior se aúna el hecho de que sólo la mitad tiene derecho a recibir atención médica en una institución pública o privada. En síntesis, los datos anteriores hacen evidente que esta vulnerabilidad de los adultos mayores se acentúa en el área rural y en el caso de las mujeres.

No obstante, a pesar de que en el país comienzan a realizarse algunos estudios de corte epidemiológico sobre el tema del envejecimiento, ${ }^{21,22}$ poco se sabe sobre cómo viven y enfrentan cotidiana y actualmente la vejez los adultos mayores y cuáles son sus principales necesidades y temores, sobre todo en el caso de la población adulta mayor que vive en condiciones de pobreza. 
Este estudio abordó las experiencias de envejecimiento de hombres y mujeres mayores de 60 años de edad a través de entrevistas semiestructuradas. La investigación se llevó a cabo en contextos rurales de los estados de Guerrero y Morelos. Se trabajó en comunidades con menos de 2500 habitantes, eminentemente campesinas, cuyos pobladores se emplean en el sector informal, en actividades marginales, por lo que son consideradas pobres y de alta marginación. Los hogares en estas comunidades se caracterizaron, en su mayoría, por tener viviendas construidas con materiales frágiles y piso de tierra, aunque algunas contaban con piso de cemento; los techos eran principalmente de teja, lámina de asbesto o de cartón. Asimismo se encontraron algunas casas de ladrillo y con techos de concreto, así como de otro tipo de materiales que iban desde carrizos hasta láminas de metal $\mathrm{u}$ otro tipo de desperdicios industriales con techos de palma o zacate de arroz. En general, contaban con dos o tres cuartos que les servían de recámara común, cocina y comedor, muchos carentes de agua potable, drenaje y de otros servicios, entre ellos los de atención médica.

\section{Material y métodos}

Se diseñó un estudio cualitativo para explorar con mayor profundidad algunos factores importantes para comprender la vivencia del envejecimiento y responder a la pregunta de investigación ¿cómo viven el envejecimiento, en contextos rurales empobrecidos, mujeres y hombres mayores de 60 años? Se partió de la idea de que la identidad de género, entendida como los roles y comportamientos culturalmente asumidos para hombres y mujeres, desempeña un papel fundamental en la configuración de la experiencia diferencial del proceso de envejecimiento al igual que la pobreza, debido a que, entre otras cosas, las oportunidades de desarrollo y las responsabilidades que de ahí se derivan promueven la desigualdad entre los sexos.

Se realizaron 11 entrevistas semiestructuradas, con base en una guía especialmente diseñada para obtener información sobre los siguientes temas: significados de la vejez; agrado o desagrado con el que se experimenta la vida en la vejez; características personales para enfrentar el proceso de envejecimiento; economía; sentido de utilidad laboral-social; experiencias de relación con miembros del grupo familiar y social; significado de la pobreza y experiencias de vida en situaciones de carencia. Se seleccionó a las personas entrevistadas en las localidades rurales de los estados de Morelos y Guerrero. El número final de entrevistas se determinó bajo el criterio de saturación teórica, entendiéndose éste como el momento en el que ya no se encontraba nueva información ${ }^{23}$ para comprender la experiencia de envejecimiento en el contexto específico. Las entrevistas fueron llevadas a cabo por estudiantes de posgrado en salud pública, previamente capacitados, en la técnica y en el manejo de la guía, por una investigadora cualitativa especializada en salud. Las entrevistas fueron grabadas, previo consentimiento informado con carta aprobada por el comité de ética del Instituto Nacional de Salud Pública y, más tarde, transcritas para su análisis en el programa informático Atlas-ti. Los grupos de análisis en cada estado diferenciaron hombres y mujeres.

\section{Resultados}

\section{Características de los participantes}

Se entrevistó a seis hombres y cinco mujeres con una media grupal de edad de 78 años (81 años para hombres y 74 para mujeres). Siete de los entrevistados vivían solos y los 11 se encontraban activos en sus comunidades, dedicados a actividades del campo, del pequeño comercio y de ayuda doméstica en hogares de terceros. En todos los casos se observó relativa funcionalidad de acuerdo con el grupo de edad: capacidad para moverse, vestirse y hacerse cargo de las necesiddes básicas cotidianas. Empero, nueve presentaban diferentes enfermedades crónicas al momento de la entrevista: diabetes mellitus, hipertensión arterial y lesiones por caídas.

Entre los principales factores relacionados con la experiencia del envejecimiento, se identificaron los siguientes: la condición de género y el rol social asignado; la condición de pobreza y de salud y la condición de compañía/ soledad y la familia. Asimismo, se identificó un componente recurrente con respecto a los principales temores asociados al envejecimiento.

\section{El género en el proceso de envejecimiento}

A través del discurso de los entrevistados, se identificó claramente que la experiencia del envejecimiento está diferenciada según la condición de género y de los roles tradicionalmente asignados, es decir, los hombres como "proveedores" y las mujeres como "cuidadoras de otros". Así, en el caso de los hombres mayores el proceso de envejecimiento se vive con mayor tristeza y frustración, en comparación con las mujeres, debido a que ellos no pueden seguir cumpliendo con el papel social y culturalmente asignado de proveer, $\mathrm{y}$ de seguir trabajando para traer sustento e ingreso al hogar: 
La tristeza es la de la vejez, que ya se siente uno menos, ya inútil, ya no es como antes..." (Hombre, Guerrero, 72 años, casado.)

La cosa es que ya no le dan trabajo a uno tan fácil, como ahorita fui a trabajar, pues no me pagaron y sin embargo uno tiene que comer, si trabaja uno [es] por necesidad." (Hombre, Morelos, 67 años, casado.)

Por su parte, para las mujeres de este contexto, si bien la experiencia del envejecimiento no es del todo agradable -en particular debido al desgaste físico, al deterioro de la salud que presentan y a las carencias económicas en las que viven-, resulta más "llevade$\mathrm{ra}^{\prime}$, ya que encuentran la posibilidad de seguir desempeñando el papel que tradicionalmente se espera de ellas, cuidando a otros.

Me siento satisfecha y le doy gracias a Dios que llegué a esta edad (...) la vejez es natural, yo fui joven, tuve mis hijos, trabajé y ahora la edad que tengo la acepto y soy feliz. (Mujer, Guerrero, 67 años, divorciada.)

Para las mujeres, cuya actividad principal ha sido cuidar a otros, el proceso del propio envejecimiento se relaciona con situaciones y experiencias más positivas: familia y compañía. En cambio, en el caso de los hombres, este proceso se asocia con sentimientos de fuerte responsabilidad y de temor de ya no poder hacerse cargo del mantenimiento de su familia. Lo anterior sugiere que la experiencia del envejecimiento les resulta menos difícil a las mujeres que siguen desempeñando su rol de cuidadoras, aunque esto está condicionado por su estado de salud y de pobreza, mientras que para los hombres es más complicado porque sienten que ya han perdido la capacidad de proveer.

... que ya no queda más que esperar la muerte porque ya no tiene uno la posibilidad de trabajar (...), entre mayor sea uno pronto se va acabando, no hay más... (Hombre, Guerrero, 76 años, casado.)

Uno de los hallazgos más interesantes de la investigación es que el mismo acontecimiento de frustración, vivido por los hombres, se repite en el caso de aquellas mujeres que, por diversas razones, también tuvieron que cumplir con el papel de proveedoras, es decir, que fueron jefas de familia:

.. que ya no puede una trabajar para ganarse el pan de cada día, yo trabajé para mantenerme toda la vida, ahorita ya no puedo (...), nunca tuve un matrimonio que hubiera sido [para] toda mi vida... (Mujer, Morelos, 68 años, madre soltera.)
¿Lo más difícil de hacerse anciana? (...) que ya no puede una trabajar. (Mujer, Morelos, 68 años, madre soltera.)

\section{La condición de pobreza y de salud-enfermedad en el envejecimiento}

A través de los testimonios de los entrevistados, se identificó que la situación de pobreza influye significativamente en la vivencia del envejecimiento. Además, que la pobreza determina en mucho la condición de salud al limitar la posibilidad de acceder a una buena alimentación, así como a tratamientos o medicamentos necesarios, de tal forma que el estado de salud sigue deteriorándose:

\section{Ahorita pienso en mi vejez (...), que ya no puedo, (...) ya no puedo hacer quehacer, no puedo hacer nada, ya ni lavar de nada... ya no puedo (...). Me duelen éstas de aquí [señalando las rodillas], mis pies me duelen, mis rodillas, todo, [señalando sus piernas y pies] pues, todo esto de aquí me duele y hay días que hasta lloro, ¿cómo, pues, voy a pasar, cómo voy a hacer para que yo me cure?, (...) no tengo [dinero] y mi hija nomás [tiene] si- quiera para comer... (Mujer, Guerrero, 62 años, sola.)}

Esta situación deteriorada del estado de salud es uno de los temores más comúnmente señalados por los ancianos entrevistados, sobre todo la condición de pérdida de independencia o movilidad; o en el caso de padecer enfermedades "largas o graves", el temor a no poder afrontarlas económicamente o a no contar con el apoyo de algún familiar para su cuidado. Así, la condición de pobreza agudiza el proceso de envejecimiento, especialmente si no se cuenta con algún tipo de ingreso estable -pensión o salario-, o con una red social de apoyo -hijos, compadres, vecinos o amigos-. En el caso de los hombres, esta situación se resiente aún más debido a la poca consolidación o existencia de redes sociales y a que muchos de ellos ya no pueden seguir trabajando como antes, mientras que las mujeres utilizan estrategias diversas para aumentar y mantener estas redes a lo largo de su vida, además de realizar trabajo doméstico y/o dedicarse al pequeño comercio para obtener ingresos. Los miembros de la familia, $y$ en particular los hijos, juegan un papel central en la vivencia del envejecimiento, en particular en el caso de quienes han enviudado:

la familia es una alegría porque pues, ¿se imagina?, uno solito pues no, se ve uno más triste, más agotado, más así [...] yo ahorita con toda mi familia me llevo bien y ellos conmigo y pues que, casi no nos visitamos pero cuando menos ahí la llevamos. (Mujer, Morelos, 75 años, viuda.) 
... pues a veces mi nieta, un hijo que tengo en el Norte, o un nieto me llegan a mandar cinco dólares... Hasta la vez no recibo más, y de ahí cuando me ven, me dan y cuando no, pues no me dan... (Hombre, Guerrero, 88 años, casado por segunda vez.)

...pues a veces me quedan 400 pesos al mes [...] y mi hija que está en el Norte en veces me va mandando 100 dólares y pues como nada más somos dos es poquito ya mi gasto... No, no me alcanza. (Mujer, Guerrero, 76 años, viuda.)

\section{La familia y la compañía durante la vejez}

En general es difícil envejecer en soledad, pero ésta parece ser una experiencia más severa para los hombres que para las mujeres, pues ellos expresan sentimientos de tristeza y depresión, y consideran que el hecho de que nadie los cuide en esa etapa de su vida es una "injusticia", puesto que trabajaron y fueron proveedores de bienes para su familia con la esperanza de estar acompañados y atendidos por alguien en su vejez. En este sentido, la vida de los hombres mayores que han enviudado y que no viven con alguno de sus hijos es especialmente difícil, ya que no son capaces de desempeñar tareas domésticas básicas como son el aseo de su hogar y la preparación de alimentos. Además, y contrariamente a lo que sucede con las mujeres, en general los hombres no tejen redes sociales a lo largo de su vida; por lo tanto, cuentan con menos recursos externos a su familia. Los apoyos más significativos y constantes provienen de los propios cónyuges o de los hijos y, ocasionalmente, de los nietos. Por último cabe destacar que, en algunos casos, son las nueras quienes se encargan de los cuidados y la alimentación de los ancianos:

... pues como ahorita estoy caminando bien con todos mis hijos y mis nueras y me quieren mucho, será porque me porto bien (...), ellas me dan de comer... (Hombre, Morelos, 100 años, viudo.)

Las redes sociales de las mujeres son más densas, efectivas, duraderas y multifuncionales, porque, finalmente, ellas las tejieron a lo largo de sus vidas y, por lo tanto, cuentan con un mayor capital humano fuera de la familia para "ir pasando" la vejez. Estas redes de apoyo incluyen a hijos, comadres, vecinas, amigas, nueras y nietos.

\section{Los significados de la vejez}

Otro hallazgo importante del estudio fue la exploración de los significados de la vejez a través del discurso de los entrevistados. Estos fueron, en general, negativos -asociados a soledad, inutilidad, deterioro y enfermedad- y estuvieron muy influenciados por la condición de pobreza, salud y género. Sin embargo, y de nueva cuenta, la visión es aún más negativa en el caso de los varones, quienes la asocian con la responsabilidad y la incapacidad de cumplir su rol tradicional, y especialmente difícil y triste en el caso de hombres viudos que viven solos. La percepción y el significado de hacerse viejo es más positiva en las mujeres, sobre todo si tienen hijos, nietos y otros familiares cercanos con quienes pueden seguir desempeñando su rol de cuidadoras. No obstante, lo anterior es difícil en el caso de las viudas que carecen de ingresos y de apoyo, o para aquellas mujeres que cumplieron el rol de proveedoras

...llegar a los 60 años (...) ya se empieza a poner difícil, claro, porque pues ya no es lo mismo para uno, para trabajar, para hacer todo lo que necesita uno hacer (...). Yo más o menos por ahí de los 60, así empecé a sentir que ya no era yo el mismo, y empieza uno a flaquear, pues aunque quiera uno, ya no puede hacer nada (...). Ahorita pues, yo por las enfermedades que tengo de las rodillas, es lo que me fregó, tuve un accidente en una camioneta y eso es lo que me dejó así, güilo. (Hombre, Guerrero, 76 años, casado.)

Cabe mencionar que, sin tomar en cuenta la condición de género, a lo largo de las entrevistas y según el análisis y la interpretación realizados, entre los adultos mayores se identificaron, como recurrentes, ciertos temores en relación con el proceso de envejecimiento. Entre los más mencionados y bien identificados están el de la soledad: estar solo en esta etapa de la vida -sentirse desprotegido- y el de morir solo -en abandono- son dos de las ideas que más angustia generan entre la población entrevistada. Estas ideas se asocian con aquella de la enfermedad, pues estar enfermo y solo es considerado como un problema real para poder desarrollar acciones o tomar decisiones acertadas a tiempo. Otro de los temores más claramente identificados es el de la pobreza: ser viejo y pobre se considera como un gran problema, porque como ya no se puede trabajar y los ingresos no suelen ser constantes ni suficientes, se debe afrontar la vida diaria casi al mínimo, ahorrando en lo que se pueda, por si hace falta posteriormente, en particular en una situación de emergencia, como una enfermedad grave o crónica. Este último factor constituye una gran preocupación para la mayoría de las personas entrevistadas, inclusive para los que gozan, en apariencia, de una mejor condición económica. Otro temor claramente identificado es el de ir perdiendo habilidades físicas e intelectuales, lo que se traduce como "pérdida de independencia".

salud pública de méxico / vol.48, no.1, enero-febrero de 2006 
Por último, en relación con los servicios, las personas entrevistadas identificaron un gran vacío en la respuesta institucional a sus necesidades. El único apoyo con el que cuentan estos ancianos se reduce al proporcionado por familiares cercanos; no se tiene la esperanza de que el gobierno o la sociedad ayuden de otra manera, aunque se sabe de la existencia de apoyos gubernamentales en otros contextos, como los urbanos. Por eso, a menudo se observa una resignación a la "voluntad divina", lo que justifica las difíciles experiencias del envejecimiento.

Cuando escucho la palabra pobre me entristezco porque yo tuve dinero, no mucho... Ahora estoy pobre, pero no pierdo la fe en Dios, siento pues que con esa fe yo vivo (Hombre, Guerrero, 88 años, casado por segunda vez.)

Doy gracias a Dios que llegué a esta edad porque cuántas no mueren jóvenes y yo ya a mis 77 años y todavía sigo dando lata... (Mujer, Guerrero, 77 años, separada.)

\section{Discusión}

\section{EI envejecimiento según la condición de género}

La aportación más importante de este estudio consistió en corroborar que la experiencia del envejecimiento es muy diferente según la condición de género, es decir, el papel o rol que tradicionalmente se espera que cumpla cada individuo en la sociedad, de acuerdo con su condición de hombre o de mujer. Así, en el caso de los varones se asume y se espera que sean ellos quienes se encarguen de proveer los bienes materiales necesarios para la reproducción del grupo familiar; mientras que para las mujeres, se asume y se espera que sean ellas quienes se ocupen de brindar cuidados al grupo familiar, diferencia que ya ha sido referida por otros estudios. ${ }^{24}$

Estas expectativas sociales se ven claramente reflejadas en los diversos testimonios de los adultos mayores que, de acuerdo con su condición de género, expresan con claridad la diferencia entre envejecer como hombres o como mujeres. En el caso de las mujeres, la experiencia del envejecimiento resulta ser, en general, más positiva, debido a que aun en esta etapa de la vida se puede seguir cumpliendo con la expectativa social de ser "cuidadora de otros". Empero, en el caso de los hombres, el papel socialmente esperado se ve muy limitado, no sólo por la edad, sino por sus restricciones físicas y problemas de salud, lo que determina en mucho su posibilidad real de seguir siendo los proveedores de la familia. Además, mientras que para las mujeres los conceptos de envejecimiento y de familia se relacionan con experiencias más positivas, en el caso de los hombres esos conceptos se asocian constantemente con la idea de responsabilidad y de tener que seguir cumpliendo con ser el sostén principal del grupo familiar hasta sus últimos días.

Lo anterior cobra mayor relevancia si se entiende que, en este contexto, el desempeño de los roles tradicionales de género es una demanda, no sólo personal sino también social o comunitaria, de manera tal que la imposibilidad de satisfacerla, además de poner en tela de juicio la propia identidad, también desestructura la forma como las personas se relacionan entre sí. Lo anterior resultaría, por llamarlo así, una forma de inadecuación cultural que trasciende a lo interpersonal y que se acentúa aún más en esta población, que ha vivido siempre asumiendo esta identidad. El acceso limitado a medios de comunicación, el apego a las tradiciones, las escasas oportunidades de trabajo y el bajo nivel de educación, son algunos factores que, en el contexto rural, dificultan la modificación de estos roles tradicionales de género. ${ }^{25,26}$ La importancia del género estriba, como lo señala Marta Lamas, ${ }^{8}$ en que orienta la percepción de lo político, lo social, lo religioso y, en general, lo cotidiano, de manera que la experiencia de vida en esta etapa no es otra cosa que el reflejo de lo aprendido a través de la cultura, con repercusiones en todos los aspectos de la vida en general.

Otro factor bien identificado a lo largo de las entrevistas son las diferencias en la experiencia de envejecer, dependiendo de si se está solo o acompañado -por la pareja y / o la familia-, especialmente en el caso de los hombres. La familia desempeña entonces un papel fundamental en la vivencia de la vejez, tal como lo señalan diversos autores. ${ }^{15,27-29}$ Esta participación por parte de la familia se vuelve necesaria en un escenario donde las acciones y apoyos formales por parte del Estado son escasos. ${ }^{30}$ Así, los casos de hombres viudos y solos, que no tienen a nadie que los cuide, suelen ser aquéllos donde se identifican los testimonios más negativos, con una carga emocional de tristeza y decepción, de sentir que la vida no "les jugó limpio al final", pues su expectativa era que una mujer los cuidara y los acompañara en sus últimos días. En cambio, para los viudos que viven con algún hijo o hija la situación a menudo es menos dolorosa porque, finalmente, existe otra mujer -hija o nuera- que se hace cargo de ellos.

La condición de género también explica la construcción diferencial de redes sociales que influye en la experiencia del envejecimiento en esta población, en particular la de parientes y amigos, pues de eso depende, en buena medida, cómo se vive esta etapa de la vida. Así, como desde el punto de vista social a las 
mujeres se les asigna la construcción de esas redes a lo largo de la vida, son ellas quienes tienen los contactos necesarios y quienes han desarrollado las habilidades para socializar con diferentes personas en la comunidad, mientras que los hombres, de quienes no se espera la construcción de estas redes, no las han tejido a lo largo de su vida y se encuentran, entonces, con un número menor de amistades y de relaciones que las mujeres. ${ }^{31-33}$ De ahí que, mientras varias de las mujeres entrevistadas narran su vida cotidiana incorporando visitas a amigas, comadres o parientes, o asistiendo a grupos diversos, la mayoría de los hombres la cuentan pasando el día más bien solos, o sentados, "esperando la muerte...".

\section{La diferencia de la experiencia del envejecimiento, según la condición de pobreza y de salud-enfermedad}

El contexto de pobreza es otro factor que condiciona la debilidad de las redes sociales de estos adultos mayores. Cattell ${ }^{34}$ refiere que la pobreza está relacionada con la privación y exclusión de las personas de los sistemas sociales, económicos, políticos y culturales que determinan su integración en la sociedad. En este mismo sentido, Campos-Navarro y colaboradores ${ }^{35}$ apuntan que los adultos mayores con menores ingresos son quienes menor oportunidad tienen de participar en los programas de apoyo, autoayuda y recreación institucionales. Esta exclusión empobrece, por tanto, las redes sociales, que en el ámbito de la salud recobran importancia porque constituyen una fuente potencial de protección para los individuos y representan una parte importante del capital social que contribuye a mejorar la calidad de vida. ${ }^{34,36}$

De esta manera, la desigualdad de género se entrelaza con la desigualdad socioeconómica y da como resultado una representación del envejecimiento en términos de pérdida, limitación y un débil estado de bienestar. Las experiencias de los adultos mayores en condiciones de vida relativamente seguras -alguna jubilación o con ingresos por algún tipo de negociose notifican como más positivas y con menor angustia y preocupación, ${ }^{37, *}$ en comparación con las personas que, además de enfrentar la vejez y posibles enfermedades, deben preocuparse por resolver la situación cotidiana de necesidades básicas como la alimentación. A esto se suma la constante angustia de pensar cómo

\footnotetext{
* Pelcastre B, Treviño S, Márquez M. Informe final del componente cualitativo del estudio "Calidad de vida y vejez en México rural: una perspectiva de género". Documento no publicado 2003.
}

se podría resolver la situación en el caso de alguna enfermedad grave o muy larga.

Las personas entrevistadas que señalaron tener una condición de salud estable, o no crítica, o sin grandes limitantes para el desarrollo de sus actividades diarias y de su independencia, son quienes narraron mejores experiencias y sentimientos asociados a la vejez, mientras que quienes indicaron tener problemas de salud constantes o que limitan de alguna manera sus actividades diarias manifiestaron mayor angustia y menos satisfacción con esta etapa de su vida.

\section{Principales temores asociados al proceso de envejecimiento}

Los temores expresados por los adultos mayores son resultado de la confrontación de lo que se considera un valor social y se convierte en expectativa en relación con lo que se ha conseguido hasta ese momento de la vida; así, aparecen la soledad frente a la red social, la enfermedad frente a la salud, la pobreza frente a la solvencia y la dependencia frente a la autonomía. Estos valores son, además, imprescindibles para vivir satisfactoriamente no sólo en la vejez, sino en cualquier etapa de la vida, considerando un estándar mínimo de calidad de vida, entendido como el resultado de las condiciones de vida, la satisfacción personal y los valores personales. ${ }^{38,39}$

\section{Conclusiones}

La identidad de género es fundamental en la configuración de la experiencia diferencial del proceso de envejecimiento, debido a que, entre otras cosas, las oportunidades de desarrollo y las responsabilidades que de ahí se derivan promueven la desigualdad entre los sexos. Envejecer, según la condición de ser hombre versus la de ser mujer, asociada al papel que tradicionalmente se le ha asignado y se espera de cada quien, es determinante en la satisfacción y experiencia que se tiene en esta etapa de la vida, lo que resulta, en términos generales, más satisfactorio para las mujeres que para los hombres.

El factor anterior se cumple o se complementa siempre y cuando se realice el papel que tradicionalmente le ha asignado la sociedad. Esto se rompe en el caso de las mujeres que deben tomar el papel de proveedoras de la familia y que notifican experiencias de vejez menos satisfactorias, en comparación con las otras mujeres que sólo debieron cumplir con el rol de cuidadoras. Así, la historia personal de cada sujeto resulta determinante para poder comprender la vivencia y experiencia de ese ciclo de la vida. 
Otro factor determinante en el proceso de envejecimiento es el del contexto específico en el cual se envejece; en este caso, rural y pobre -falta de servicios, infraestructura, etcétera-. Envejecer en un contexto específico como éste determina, en gran medida, cómo se vive y se experimenta la vejez.

Un factor recurrente a lo largo de la investigación es el del sentimiento de soledad con el que se vive en buena medida el proceso del envejecimiento y que amerita un mayor análisis, pues es uno de los principales temores de la propia población entrevistada y, de hecho en el campo, resulta palpable la situación de abandono y soledad que prevalece en varios casos. Lo anterior pudiera explicar, tal vez, la gran importancia de los testimonios religiosos y el determinismo divino como una especie de justificación de la manera como se vive esta etapa de la vida.

Entre los principales temores comunicados por la población adulta mayor está la gran ausencia de respuesta institucional, más que la social, que se ofrece en estos contextos rurales y de pobreza, para atenderlos de alguna manera. Como investigadores y prestadores de servicios de salud este punto debería ser determinante, debido al crecimiento esperado de la población adulta mayor durante las próximas décadas.

Finalmente, es necesario tomar en consideración algunas cuestiones para la investigación y la acción futuras, como por ejemplo fomentar la investigación en este grupo de edad para comprender el proceso de envejecimiento en diversos contextos y en las distintas entidades del país; sensibilizar a los tomadores de decisiones para que se realicen acciones tomando en cuenta las necesidades de este grupo de edad, de acuerdo con la condición de género y el contexto específico (rural/ urbano); preparar cuadros profesionales para dar respuesta a las demandas derivadas del crecimiento de este grupo poblacional y desarrollar una cultura de respeto a los adultos mayores, por medio de campañas masivas de sensibilización a la población en general.

\section{Referencias}

I. Foro Mundial ONG sobre Envejecimiento. Propuestas y recomendaciones de acción para los gobiernos y la sociedad civil. Madrid, 5-9 abril 2002: I-4. Disponible en: http://www.forumageing.org/ espanol/informacion/propuestas.htm.

2. Partida V. Perspectivas del cambio demográfico. Monto y estructura de la población en el año 2000 y perspectivas en el 2050. Demos 200I: 6-7. 3. Fraga JC, Mena M. Envejecimiento. Un reto adicional. Apuntes para su estudio en América Latina. En: Bronfman M, Castro R, coords. Salud, cambio social y política. Perspectivas desde América Latina. México, D. F.: EDAMEX, 1999: 44I-464.
4. Wong R. Transferencias intrafamiliares e intergeneracionales en México. En: Envejecimiento demográfico en México: retos y perspectivas. México, D. F.: CONAPO, 1999: I47-I69.

5. Ortega MT. Educación de la mujer indígena: estrategia de desarrollo regional. En: Galeana P, comp. La condición de la mujer indígena y sus derechos fundamentales. México, D. F.: UNAM-Coordinación de Humanidades, 1997: 103-116.

6. Lara MA. Inventario de masculinidad y feminidad (IMAFE). México, D.F.: El Manual Moderno, 1993.

7. Montes de Oca V. Diferencias de género en el sistema de apoyo a la población envejecida en México. Papeles Poblac 1999; (19): 149-172. 8. Lamas M. Cuerpo: Diferencia sexual y género. México, D. F.:Taurus, 2002. 9. INEGI. XII Censo General de Población y Vivienda. México, D. F., 2000. 10. Damián A, Boltvinik J. Evolución y características de la pobreza en México. Comercio exterior 2003; 53 (6): 519-531.

II. Boltvinik J, Hernández E. Pobreza y distribución del ingreso en México. México, D. F:: Siglo XXI, 1999.

12. Cortés $F$. Consideraciones sobre la marginalidad, marginación, pobreza, y desigualdad en la distribución del ingreso. Papeles Poblac 31; México: Centro de Investigación y Estudio de la Población/Universidad Autónoma del Estado de México, 2002. Disponible en: http:// papelesdepoblacion.uaemex.mx/rev31/pdf/cortes3l.pdf 13. Barrón L, Trejo G. La pobreza en México: la paradoja de la política social. En: Rubio L, Fernández A, eds. México a la hora del cambio. México: Cal y Arena/CID, 1995. Disponible en: http://www.cidac.org/ libroscidac/mexico-cambio/Cap-16.PDF.

I4. IFAD. IFAD Seminar on Rural Poverty Reduction "Harvesting IFADBSF Synergies". Report, 2002. Bruselas: IFAD.

15. Montes de Oca V. Familia, sociedad y vejez. Las personas adultas mayores y sus apoyos informales. Demos 200I: 34-35.

16. Aparicio R. Transición demográfica y vulnerabilidad durante la vejez. En: La situación demográfica de México. México, D. F.: CONAPO, 2003: I55-168.

17. Gomes de Conceiçao MC. Condiciones socioeconómicas de la tercera edad. Demos 200I: I3-I5.

18. Wong R, Espinoza M. Ingreso y bienes de la población de edad media y avanzada en México, Papeles Poblac 37, México, DF: CIEAP/UAEM 2003: I29-I66.

19. Ham-Chande R. Características sociales y económicas en las edades avanzadas. En: El envejecimiento en México: el siguiente reto de la transición demográfica. México: El Colegio de la Frontera Norte, 2003: I7I-216.

20. Welti-Chanes C. Economía y envejecimiento. Cambios socioeconómicos y sobrevivencia de la población mayor. Demos 2001: 25-26.

21. Salgado N,Wong R, ed. Envejeciendo en la pobreza. Género, salud y calidad de vida. México, D.F.: Instituto Nacional de Salud Pública, 2003.

22. Demos. Número especial sobre envejecimiento. México, D. F.: UNAM- Instituto de Investigaciones Sociales, 2001.

23. Castro R. En busca del significado: supuestos, alcances y limitaciones del análisis cualitativo. En: Szasz I, Lerner S, eds. Para comprender la subjetividad. Investigación cualitativa en salud reproductiva y sexualidad. México, D. F.: El Colegio de México, 1996: 57-85.

24. Monchietti A, Caballero I, Sánchez M, Lombardo E. Representaciones de la vejez. Rev Latinoam Psicol 2000; 32 (3): 519-536.

25. Mier y Terán M. Pobreza y transiciones familiares a la vida adulta en las localidades rurales de la península de Yucatán. Población y Salud en Mesoamérica. Revista electrónica 2004; 2(I) artículo 5: I-43. Disponible en: http://ccp.ucr.ac.cr/revista

26. Montes de Oca V. Redes comunitarias, género y envejecimiento, Cuadernos de Investigación 3I, México, D. F.: UNAM- Instituto de Investigaciones Sociales, 2005: 16I. 
27. Ham-Chande R. Vejez y dependencia. Paradigmas y nuevos contratos sociales. Demos 200I: 27-28.

28. Palma Y. La gran metrópoli y los viejos. La población mayor en la zona metropolitana de la Ciudad de México. Demos 2001:36-37.

29. Palma JL. El apoyo familiar. Transferencias de y para la población mayor dentro y fuera del hogar. Demos 200I: 40-4l.

30. Olvera L. Fracasan políticas estatales de atención a la tercera edad. Gaceta UNAM 2004, (3698).

31. Contreras de Lehr E.Ageing and family support in Mexico. En: Kending HL, Hashimoto A, Coppard LC, comps. Family support for the elderly: The international experience. Nueva York: Oxford University Press, 1992: 215-223.

32.Varley A, Blasco M. ¿Cosechan lo que siembran? Mujeres ancianas, vivienda y relaciones familiares en el México urbano. En: Gomes $C$, comp. Procesos sociales, población y familia. Alternativas teóricas y empíricas en las investigaciones sobre vida doméstica. México, D.F: FLACSO/Porrúa, 200I: 30I-322.

33. Montes de Oca V. Diferencias de género en el sistema de apoyo a la población envejecida en México. Papeles Poblac 19; México: Centro de Investigación y Estudios de la Población / Universidad Autónoma del Estado de México, 1999: 149-172.
34. Cattell V. Poor people, poor places, and poor health: The mediating role of social networks and social capital. Soc Sci Med 200I; (52): I502-1516.

35. Campos-Navarro R, Torrez D, Arganis-Juárez E. Las representaciones del padecer en ancianos con enfermedades crónicas. Un estudio en la Ciudad de México. Cad Saude Publica 2002; 18 (5): I27I-I279.

36. Sluzki C. De cómo la red social afecta la salud del individuo y la salud del individuo afecta la red social. En: La red social: frontera de la práctica sistémica. Barcelona: Gedisa, 1996: 71-91.

37. Wong R. La relación entre salud y nivel socioeconómico entre adultos mayores: diferencias por género. En: Salgado N, Wong R, eds. Envejeciendo en la pobreza. Género, salud y calidad de vida. México, D.F:: Instituto Nacional de Salud Pública, 2003: 97-I 22.

38. Borthwick-Duffy SA. Quality of life and quality of care in mental retardation. En: Rowitz L, ed. Mental retardation in the year 2000. Berlin: Springer-Verlag, 1992: 52-66.

39. Felce D, Perry J. Quality of life: It's definition and measurement. Res Dev Disabil 1995; I6 (I): 5I-74. 